\title{
INSIGHTS
}

\section{Family reflections on phenylketonuria}

\author{
Christine S. Brown ${ }^{1}$ \\ Pediatric Research (2018) 84:797-798; https://doi.org/10.1038/s41390-018-0179-5
}

I am writing this commentary as both a parent of two children with phenylketonuria (PKU) and as a professional as the Executive Director of the National PKU Alliance, a nonprofit patient advocacy organization working to improve the lives of people with PKU and pursue a cure.

Most medical professionals know of PKU as a metabolic disorder that is diagnosed through newborn screening. People living with PKU cannot process the amino acid called phenylalanine, found in all natural sources of protein, due to a deficiency in the phenylalanine hydroxylase enzyme. This enzyme converts phenylalanine into tyrosine, another essential amino acid. In turn, tyrosine is important in the production of many neurotransmitters, such as dopamine and serotonin. For decades, professionals and the patient community have discussed PKU as a dietary condition because treatment has centered around medical foods and a restrictive diet very low in protein. However, in essence, PKU is a rare brain threatening disorder as excess phenylalanine is toxic to the brain. Every piece of food that I give to my children to eat determines the health of their brain and their intellectual outcomes.

I am extremely fortunate and blessed that my children were diagnosed with PKU at birth through newborn screening. I am lucky as $2 / 3$ of newborn babies worldwide have no access to newborn screening. That means one baby every hour is born in this world with PKU that is not diagnosed. Both of my boys treat their PKU with medical foods and a restrictive diet. Again-I am one of the fortunate ones. My boys receive their medical foods directly from the state we live in, so I do not have to deal with routine insurance denials for their medical foods treatment that prevents severe intellectual disability. While 39 states have some sort of law mandating coverage for medical foods to treat PKU, many of them have loopholes and age limits even though PKU must be treated for life. The NPKUA is trying to change this with the Medical Foods Nutrition Equity Act (S. 1194 and H.R. 2587), a bill before Congress that would require all public and private health insurance programs to cover medical foods for the treatment of inherited metabolic disorders and certain GI conditions with no age limits.

However, treating PKU with medical foods is not simply just a diet. I don't know of any other approved therapy for a disease that is so isolating on a daily basis as dietary therapy. People with PKU are severely restricted in their everyday life as eating is how we socialize, celebrate, explore, and form friendships and relationships. My boys can only eat $6 \mathrm{~g}$ of natural protein a day. They cannot eat anything with meat, soy, dairy, nuts, legumes, wheat, or flour. What do you do when you travel to a new country to experience a new culture? You eat their food. What do you have to celebrate your birthday? Cake. What did my son's seventh grade football team do to celebrate an undefeated season? A pizza party. How do you celebrate Thanksgiving? Turkey. What do you do on a first date? Go out for dinner. These experiences are not part of my children's world because every single thing they put into their mouth has to be weighed, measured, and calculated. Every single thing they put in their mouth has the potential to cause harm to their brain. And now, as they get older, their PKU is getting more difficult to manage with the tools I have available.

Dietary treatment fails the majority of even the most engaged people with PKU. Two years ago, the NPKUA finalized its first patient survey to see what the community wanted in terms of new treatments for PKU. A total of 625 subjects responded to the survey. Close to $62 \%$ adults and $26 \%$ of children reported average blood phe levels above the American College of Medical Genetics and Genomics recommended range of $360 \mu \mathrm{mol} / \mathrm{L}$; and, these are the engaged PKU patients. The vast majority that filled out the survey had visited their PKU clinic in the last year and tested their phenylalanine levels on a monthly basis. Respondents overwhelmingly know what is needed to manage their PKU and have access to care in a clinic setting. However, challenges to adhering to dietary therapy was evident.

$91 \%$ of everyone surveyed reported that the development of new treatments was important to them. The tools we have available now-medical foods and dietary therapy and Kuvan used in conjunction with dietary therapy simply do not work in the majority of our community to adequately reduce phenylalanine levels. Palynziq, an enzyme substitution therapy recently approved by the FDA in adults, does give us hope. But, we want and deserve more.

We now know through NPKUA funded research that there are abnormalities in both the white and gray matter of the brains in people with PKU. The white matter integrity is compromised even in individuals with well-controlled diets. In addition, new research shows differences in the gray matter structures (Anderson \& Leuzzi, MGM, 2010 and Christ et al, JIMD, 2012). Even with early and continuous treatment, individuals with PKU experience cognitive and neurologic disruptions, including a slight decrease overall in IQ and impairments in executive function, processing speed, and emotional regulation (Bilder et al, Developmental Neuropsychology, 2016). In addition, many well-controlled children display a variety of disorders, including anxiety, ADHD, and depression (Antshel \& Waisbren, Neuropsychology, 2003).

Our community deserves new treatments so children and adults can achieve lower and even normal levels of phenylalanine. We want treatments that allow an unrestricted diet and address the symptoms of PKU. According to the NPKUA patient

${ }^{1}$ National PKU Alliance, Inc., Eau Claire, USA

Correspondence: Christine S. Brown (info@pedres.org)

Received: 5 September 2018 Accepted: 5 September 2018

Published online: 13 September 2018 
survey, the overwhelmingly majority of PKU patients want treatments that reduce their blood phenylalanine concentrations, improve their attention span and ability to focus, improve executive function skills and decrease depression and anxiety. While medical foods have successfully reduced the most severe manifestations of PKU, I don't want my boys to grow up to just be "good enough." They deserve to be the best they can be and have optimal outcomes. No parent would settle for anything less.

Christine S. Brown 